\title{
Stabilizing a system with an unbounded random gain using only finitely many bits
}

\author{
Victoria Kostina*, Yuval Peres, Gireeja Ranade ${ }^{\dagger}$, Mark Sellke \\ ${ }^{*}$ California Institute of Technology, Pasadena, CA \\ $\dagger$ University of California, Berkeley \\ $\ddagger$ Stanford University \\ vkostina@caltech.edu, yuval@yuvalperes.com,ranade@eecs.berkeley.edu, msellke@stanford.edu
}

\begin{abstract}
We study the stabilization of a linear control system with an unbounded random system gain where the controller must act based on a rate-limited observation of the state. More precisely, we consider the system $X_{n+1}=A_{n} X_{n}+W_{n}-U_{n}$, where the $A_{n}$ 's are drawn independently at random at each time $n$ from a known distribution with unbounded support, and where the controller receives at most $R$ bits about the system state at each time from an encoder. We provide a time-varying achievable strategy to stabilize the system in a second-moment sense with fixed, finite $R$.

While our previous result provided a strategy to stabilize this system using a variable-rate code, this work provides an achievable strategy using a fixed-rate code. The strategy we employ to achieve this is time-varying and takes different actions depending on the value of the state. It proceeds in two modes: a normal mode (or zoom-in), where the realization of $A_{n}$ is typical, and an emergency mode (or zoom-out), where the realization of $A_{n}$ is exceptionally large. To analyze the performance of the scheme we construct an auxiliary sequence that bounds the state $X_{n}$, and then bound auxiliary sequence in both the zoom-in and zoom-out modes.
\end{abstract}

\section{INTRODUCTION}

System design for decentralized control over communication networks requires an understanding of the informational bottlenecks that affect our ability to stabilize the system. This paper focuses on a system that grows unpredictably (i.e. has an unbounded random system gain) and is observed over a ratelimited channel. We consider a modification of the classical data-rate theorems [2], [3], [4], [5], where the system growth is unpredictable and random, and provide a strategy to control such a system over finite and fixed-rate channel. This builds on previous work [6], which considered the control of a system with unpredictable growth with unbounded support over a channel using a variable-rate code.

Specifically, we consider the control of the following system:

$$
X_{n+1}=A_{n} X_{n}+W_{n}-U_{n}
$$

Here $X_{n}$ is the (scalar) state of the system at time $n$. The system gains $\left\{A_{n}\right\}_{n \geq 0}$ are drawn i.i.d. from a known distribution

This paper was previously presented in part at ISIT 2018 [1]. Copyright (c) 2020 IEEE. Personal use of this material is permitted.

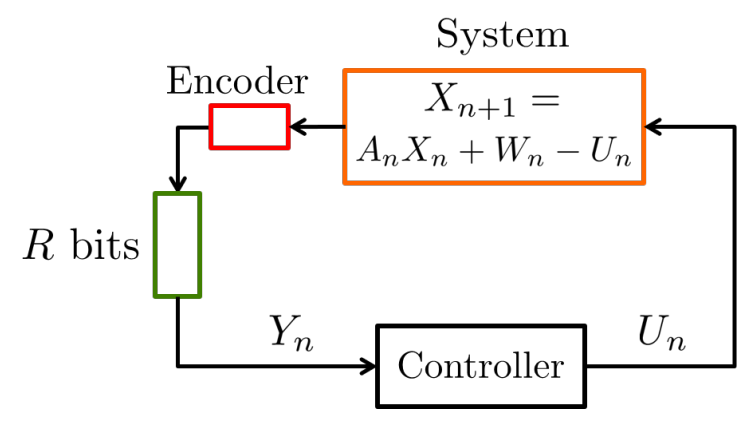

Fig. 1. Rate-limited control: The control $U_{n}$ can be any function of the observations $Y_{0}^{n}$. The encoder chooses exactly $R$ bits to transmit to the controller at every time-step through $Y_{n}$, i.e. $Y_{n} \in\left\{1,2, \ldots 2^{R}\right\}$. The system gains $\left\{A_{n}\right\}_{n \geq 0}$ are i.i.d. random variables, and so are the additive disturbances $\left\{W_{n}\right\}_{n \geq 0}$.

and model the uncertainty the controller has about the system. The additive disturbances $\left\{W_{n}\right\}_{n \geq 0}$ are also i.i.d. drawn from a known distribution. The controller chooses the control $U_{n}$ causally based on $R$-bit observations $Y_{0}^{n}:=\left\{Y_{0}, \ldots, Y_{n}\right\}$, where $Y_{n} \in\left\{1,2, \ldots 2^{R}\right\}$, which are transmitted by an encoder co-located with the system. Our goal is to stabilize the system in a second-moment sense, which thus requires the co-design of an encoder-controller pair.

It is known that without a rate-limit on the channel, the system in (1) is second-moment stabilizable if and only if $\sigma_{A}^{2}<1$, where $\sigma_{A}^{2}$ is the variance of $A_{n}$ [7]. Hence, we focus on the case $\sigma_{A}^{2}<1$. The goal is to guarantee second-moment stability of the system. Our main theorem is stated below.

Theorem 1.1. Assume that $A_{n}$ and $W_{n}$ have finite $(4+\epsilon)$ th moments. Then, for some $R \in \mathbb{N}$, there exists a rate- $R$ limited control strategy (see Fig. 1) that achieves secondmoment stability, i.e.,

$$
\limsup _{n \rightarrow \infty} \mathbb{E}\left[X_{n}^{2}\right]<\infty .
$$

\section{A. Strategy overview}

Previous works (e.g. [8], [9], [6]) have investigated variablerate coding strategies for stabilizing the second-moment of the system (1), as well as other related systems. However, variablerate strategies fundamentally require that the encoder send an unbounded number of bits in the case when the realization 
of $A_{n}$ is large, and cannot be directly adapted for use in a fixed-rate regime.

In contrast to the variable-rate strategies, we employ a time-varying strategy in this work. It works in two different "modes," depending on whether the realization of $A_{n}$ was typical or exceptionally large. We will describe the strategy precisely later, but provide an intuitive description here.

At every time-step, the controller maintains an interval that estimates system state. When the state remains in the interval that was estimated by the controller, the strategy proceeds in "normal" (zoom-in) mode. If the state escapes the predicted interval, usually due to the realization of an exceptionally large $A_{n}$, the strategy enters "emergency" (zoom-out) mode. There, the controller grows it's guess for the interval at every time-step till we are able to locate $X_{n}$, following which we step back into normal mode. This time-varying strategy works because we can guarantee some average decrease during normal mode, while we can bound the blow-ups from emergency mode (since we assume that the $(4+\epsilon)$-th moments of $A$ and $W$ are bounded).

\section{B. Related work}

This paper builds on many previous ideas in information theory and control. Yüksel and Basar provide a detailed discussion focused on information structures and stabilization in their book [10]. A recently released book by Fang et al. [11] provides some more of the history as well as recently developments on the work to integrate information theory with control, with a focus on performance-limits from the inputoutput perspective represented by Bode's integral formula.

Fundamentally, our problem setup grows out of the uncertainty threshold principle [7] and the extensive work to understand data-rate theorems [2], [3], [4], [5], [12], [13], [14], [15]. While the work on the uncertainty threshold principle [7] does not consider rate limits on the observations, the classical data-rate theorems assume that the system model and growth rate are always perfectly known to the controller. Our paper jointly explores parameter uncertainty and rate-limited control.

The data-rate theorems ask the question: how does limited communication affect our ability to control a system? The results tell us that a noiseless observation data rate $R>\log |a|$ is necessary and sufficient to stabilize a system in the secondmoment sense under a few different considerations on the additive noise in the system. Matveev and Savkin [16] have further provided data-rate style theorems for continuous time systems and for different notions of stability, such as stability in probability.

Previous works have generalized the data-rate theorems for systems with additive noise to the case when the channelrate is uncertain and given by a Markov process, e.g. You and Xie [17] and Minero et al. [18]. A related body of work considers packet-drop networks, where again, a randomness is introduced in the observation process [19], [20], [21], [22]; a detailed survey of this work is provided by [23], [24]. In contrast, our paper examines whether the system can be stabilized with a fixed-rate $R$ when the growth of the system through $A_{n}$ is random.
Martins et al. [8] were the first to consider the rate-limited control of a system with uncertain growth. Their achievable scheme is quantization-based and assumes that the uncertainty on the system growth is bounded above. Phat et al. [25] also consider rate-limited control with uncertain parameters from a robust control perspective. Their setup differs from ours in that it is not stochastic, and only considers bounded support for the uncertainty on the parameters. They provide a uniform quantization scheme that can stabilize their system.

Okano and Ishii have made significant progress on understanding rate-limited control of systems with unpredictable growth from a worst-case perspective [26], [27], [9]. They consider systems where the uncertain parameters have bounded support and do not consider additive noise in their model. The achievable scheme in [9] proposes a non-uniform optimal quantizer for their problem that uses bins with logarithmically decreasing lengths, with the bins closest to zero being the largest. However, this cannot work in the setting where both $A_{n}$ and $W_{n}$ can have unbounded support, as is the case in our work. Our current paper shows that even with unbounded uncertainty on $A_{n}$ and $W_{n}$ a fixed-rate scheme can be used to stabilize the system.

In the context of linear systems with known parameters and unbounded additive disturbances, the necessity of adaptive quantization for stabilization has been long recognized. Nair and Evans [28] proved that time-invariant fixed-rate quantizers are unable to attain bounded cost if the noise is unbounded [28], regardless of their rate. The reason is that since the noise is unbounded, over time, a large magnitude noise realization will inevitably be encountered, and the dynamic range of the quantizer will be exceeded by a large margin, not permitting recovery. This necessitates the use of adaptive quantizers of the zoom-in-zoom-out type like those originally proposed by Brockett and Liberzon [29], and further studied in [28], [13], [30], [31]. Such quantizers "zoom out" (i.e. expand their quantization intervals; this corresponds to our "emergency mode") when the system is far from the target and "zoom in" when the system is close to the target (this corresponds to our "normal mode"). This paper uses this key idea of zoomin-zoom-out quantizers from the previous works and extends our recent analysis [31] of adaptive quantization for known linear systems to unpredictably varying ones. Our paper was partially presented at ISIT 2018 [1].

In this work, we take a stochastic control approach, which complements the investigations around parameter uncertainty in the robust control literature [32], [33]. We build on a series of investigations around model unpredictability and multiplicative noise in control systems [34], [35], [36].

\section{SeTUP}

We consider the system in (1). Here $W_{n}, A_{n}$ are i.i.d. variable noises drawn from the laws $W$ and $A$. These are independent of the system state $X_{n}$ and the control term $U_{n}$. We use $n$ as the time index. Without loss of generality, we assume our initial condition $X_{0}=0$. The encoder and controller will work together to stabilize the sequence $\left(X_{n}\right)$ in the second-moment sense, i.e., (2). The encoder observes $X_{n}$ 
perfectly, and transmits a symbol from the set $\left\{1,2, \ldots, 2^{R}\right\}$ to the controller at each time-step. $R$ is a finite universal constant that we will choose later. The controller can choose $U_{n}$ as a function of these $R$ bits and any previous history. Let $\mu_{A}, \sigma_{A}, \mu_{W}, \sigma_{W}$ be the means and standard deviations of $A, W$, and assume $\mu_{a} \neq 0$. Without loss of generality, set $\mu_{W}=0$.

The goal of second-moment stability cannot be accomplished for arbitrary distributions $A, W$. Using the independence of $A_{n}, W_{n}$ and $U_{n}$, we see from (1) that [7]:

$$
\mathbb{E}\left[X_{n+1}^{2}\right]=\sigma_{A}^{2} \mathbb{E}\left[X_{n}^{2}\right]+\mathbb{E}\left[\left(\mu_{A} X_{n}-U_{n}\right)^{2}\right]+\sigma_{W}^{2} .
$$

It is clear from (3) that the assumption $\sigma_{A}<1$ is required for second-moment stabilizability (except the case $\sigma_{A}=1, \sigma_{W}=$ 0 which we ignore.) Our result will provide a strategy to achieve it. We require the mild assumption that $A$ and $W$ have finite $4+\varepsilon$-moment for some $\varepsilon>0$.

\section{Description of The StRATEGy}

Before describing the strategy, we first describe what happens at every time step. At the $n$th time-step, three things happen in the following order:

1) The encoder transmits a codeword from a codebook of size $R$ bits to the controller based on the observation of $X_{n}$. The exact value of $R$ is described below.

2) The controller chooses $U_{n}$ based on the transmission from the encoder and any past history.

3) The noises $A_{n}, W_{n}$ are generated and the new state $X_{n+1}$ is determined, based on $A_{n}, W_{n}$ and $U_{n}$. This value of $X_{n+1}$ is observed by the encoder.

We proceed in "rounds," which are blocks of time-steps. Most rounds will consist of a single time-step, in those cases where $X_{n}$ falls in an interval predicted by the encodercontroller pair. In those cases where $X_{n}$ falls outside those bounds, a round might consist of multiple time-steps, until an appropriate bounding interval is found for the state.

We will maintain positive numbers $I_{n} \leq M_{n}$, that will be defined below. Roughly speaking, $M_{n}$ will represent an estimate of the maximum value of $\left|X_{n}\right|$, and $I_{n}$ will capture the quantization error in the controller's estimate of $X_{n}$.

We fix $M_{0}$ (a lower bound for the $M_{n}$ 's), a large constant $P$, and a small $\delta$ to be chosen later, such that $\frac{1}{\delta} \in \mathbb{Z}$. The constant $P$ captures the controller's guess on how the $M_{n}$ 's will grow at each time-step. The constant $\delta$ specifies the size of the codebook to $\frac{2}{\delta}+1$, and the transmission rate is $R=$ $\left\lceil\log \left(\frac{2}{\delta}+1\right)\right\rceil$.

We will now describe how the $M_{n}$ 's will evolve with time along with our strategy. We first initialize $M_{1}=I_{1}=M_{0}$. At each time-step, we check how $X_{n}$ compares to the guess of the controller. If $\left|X_{n}\right| \leq P M_{n-1}$, we operate in normal mode. In this case, the encoder transmits a quantized version of the state to the controller. If $\left|X_{n}\right|>P M_{n-1}$ we operate in emergency mode, and the encoder simply sends a special codeword to indicate this emergency mode. A round of time-steps will continue until we exit emergency mode at a time $\ell$ such that $X_{\ell} \leq P M_{\ell-1}$.
Case 1: $X_{n} \leq P M_{n-1}$.

In this case, $X_{n}$ has landed within the bounds we expected it to land in, and we are starting the round in normal mode. The encoder partitions the interval $\left[-P M_{n-1}, P M_{n-1}\right]$ into $\frac{2}{\delta}$ subintervals of length $\delta P M_{n-1}$ and sends a codeword to represent the index of the interval $\left(a_{n}, b_{n}\right)$ containing $X_{n}$. Set

$$
\begin{aligned}
M_{n} & \triangleq \max \left(M_{0},\left|a_{n}\right|,\left|b_{n}\right|\right), \\
I_{n} & \triangleq \max \left(M_{0}, \frac{\left|a_{n}-b_{n}\right|}{2}\right), \\
\rho_{n+1} & \triangleq \operatorname{sgn}\left(a_{n}\right)=\operatorname{sgn}\left(b_{n}\right),
\end{aligned}
$$

where equality in (6) holds because $\frac{1}{\delta}$ is an integer, and so the point 0 is always an endpoint of a quantization interval. The values $M_{n}, I_{n}, \rho_{n}$ are common knowledge between the encoder and controller; they are uniquely determined by the bits that have been sent by the encoder. Consequently, in normal mode, the controller knows which interval of the form

$$
\rho_{n}\left[M_{n}-2 I_{n}, M_{n}\right]
$$

contains $X_{n}$, where $\rho_{n} \in\{ \pm 1\}$.

Now the controller will set

$$
U_{n}=\rho_{n} \mu_{A}\left(M_{n}-I_{n}\right) .
$$

The random variables $A_{n}, W_{n}$ are generated, and $X_{n+1}$ is determined. Hence, we will have that:

$$
\left|\mu_{A} X_{n}-U_{n}\right| \leq \mu_{A} I_{n}
$$

If $\left|X_{n+1}\right| \leq P M_{n}$, the round ends at time-step $n+1$. Else, it will proceed for at least one more time-step.

Case 2: $\left|X_{n}\right|>P M_{n-1}$.

If this is the case, the encoder simply uses the special $\left(\frac{2}{\delta}+1-\right.$ th) codeword to indicate that we are in emergency mode and the state has escaped the predicted interval. As a result, we do not define $I_{n}$ in emergency mode. The round will continue until we exit emergency mode.

Throughout emergency mode, we will take

$$
M_{n+k+1}=P M_{n+k}
$$

until exiting. More specifically, if at the start of time-step $n+k$ we are in emergency mode, the following steps happen:

1) The encoder uses the special codeword to indicate that we are in emergency mode.

2) The controller sets $U_{n+k}=0$. Since the controller does not have a good estimate of the state, it does nothing.

3) $A_{n+k}, W_{n+k}$ are generated, and $X_{n+k+1}$ is determined. If $X_{n+k+1}<P M_{n+k}$, we exit emergency mode and the round ends. Parameters $I_{n+k+1}$ and $\rho_{n+k+1}$ are defined as in Case 1 at the start of the next normal round. Else, we continue to the next time-step in emergency mode.

Remark 3.1. Note that the state always belongs to the interval indicated by (7) at the end of a round. We also see that $M_{n}, I_{n} \geq M_{0}$ at all times. 


\section{Preparations for Analysis}

\section{A. A Modified Sequence}

The idea is to show that $\left|X_{n}\right|^{2}$ decreases on average when it is large. However this is not true during emergency mode, so we will define a dominating sequence $\left(N_{n}\right)$ which does have the desired property. The analysis will also use a device of modifying the sequence $\left(X_{n}\right)$ slightly. We will freeze the value of $X_{n}$ at a time of interest $n_{0}$, by setting the subsequent $A_{n}$ 's to 1 and $W_{n}$ 's to 0 . This modification is an important step required to obtain the dominating sequence though Proposition 4.1 as explained in Remark 4.2. If we continued to let the state $X_{n}$ evolve, then we would have to account for the probability that $X_{n_{0}}$ was large but $A_{n_{0}}$ was small.

First, we make a few important definitions. Fix $n_{0}$, where $\mathbb{E}\left[X_{n_{0}}^{2}\right]$ is what we would like to bound. We define a modified sequence as follows:

$$
\tilde{X}_{n} \triangleq \begin{cases}X_{n} & \text { if } n<n_{0}, \\ X_{n_{0}} & \text { if } n \geq n_{0} .\end{cases}
$$

which satisfies the recurrence

$$
\tilde{X}_{n+1}=\tilde{A}_{n} \tilde{X}_{n}+\tilde{W}_{n}-\tilde{U}_{n}
$$

for

$$
\left(\tilde{A}_{n}, \tilde{W}_{n}, \tilde{U}_{n}\right) \triangleq \begin{cases}\left(A_{n}, W_{n}, U_{n}\right) & \text { if } n<n_{0} \\ (1,0,0) & \text { if } n \geq n_{0}\end{cases}
$$

Define a sequence $\tilde{M}_{n}$ :

$$
\tilde{M}_{n} \triangleq \begin{cases}M_{n} & \text { if } n \leq n_{0}, \\ P \tilde{M}_{n-1} & \text { if } n>n_{0} \text { and }\left|\tilde{X}_{n_{0}}\right|>\tilde{M}_{n-1}, \\ \tilde{M}_{n-1} & \text { if } n>n_{0} \text { and }\left|\tilde{X}_{n_{0}}\right| \leq \tilde{M}_{n-1} .\end{cases}
$$

Note that $\tilde{M}_{n}$ might still be growing for $n>n_{0}$, even though the sequence $\tilde{X}_{n}$ it aims to bound is not growing for $n>n_{0}$. Finally define:

$$
\tilde{I}_{n} \triangleq \begin{cases}I_{n} & \text { if } n \leq n_{0} \\ I_{n_{0}} & \text { if } n>n_{0}\end{cases}
$$

Now we define a counter for the length of a round for the modified sequence $\tilde{X}_{n}$.

Define $\tau(n)$ by

$$
\tau(n) \triangleq \min \left\{m \geq n:\left|\tilde{X}_{m}\right| \leq P \tilde{M}_{m-1}\right\} .
$$

Assuming time-step $n<n_{0}$ does not involve an emergency which lasts until time $n_{0}$, the value $\tau(n)$ is simply the first time-step $n$ or larger which starts in normal mode. Hence for a time-step $n$ which exits in normal mode, $\tau(n)=n$. For a time-step $n$ which starts in emergency mode, suppose that time-steps $n, \ldots, n+k<n_{0}$ exit in emergency mode and timestep $n+k+1$ does not. Then

$$
\tau(n)=\tau(n+1)=\ldots=\tau(n+k)=n+k+1 .
$$

Note that we always have $\tau(\tau(n))=\tau(n) \geq n$, usually with equality.
Let $K$ be a large constant. Define

$$
Q_{n} \triangleq \sqrt{\tilde{M}_{n}^{2}+K \tilde{I}_{n}^{2}}
$$

The random sequence $Q_{n}$ is essentially $M_{n}$ but with better expected-decrease guarantees that will be relevant later.

Finally, we define the dominating sequence which we will show is decreasing on average for large $n$. For a time-step $n$, let

$$
N_{n} \triangleq Q_{\tau(n)} 2^{\tau(n)-n} .
$$

With this definition of $N_{n}$ we "front-load" the cost of an emergency mode. Thus, $N_{n}$ is decaying exponentially during the emergency mode, even though $X_{n}$ is not. This is because from (16) we know that that $\tau(n)=\tau(n+1)=\ldots=$ $\tau(n+k)=n+k+1$ if $n+k$ is the last step that exits in emergency mode. This helps us obtain the required bound. The sequence $N_{n}$ measures the time until the end of the round from any $n$ through the exponent $\tau(n)-n$. The idea is to show that $N_{n}$ decreases on average when it is large, and to conclude from this that $\mathbb{E}\left[N_{n}^{2}\right]$ is uniformly bounded. We show in Proposition 4.1 below that $N_{n_{0}}$ bounds $X_{n_{0}}$, allowing us to conclude that $\mathbb{E}\left[X_{n_{0}}^{2}\right]$ is bounded, for all $n_{0}$. This idea is similar to the one we used in the analysis of a quantized linear system with constant $A_{n}$ in [31].

For technical reasons explained just below, we will work with the sequence $\tilde{X}_{n}$ when estimating $\mathbb{E}\left[X_{n_{0}}^{2}\right]$. We think of this modified sequence as evolving normally until time $n_{0}$. At time $n_{0}$, we freeze the value of $X_{n}$ to be $X_{n_{0}}$. We finish the current round if we are in the middle of emergency mode, and then stop permanently.

Proposition 4.1. We always have

$$
\left|X_{n_{0}}\right| \leq N_{n_{0}} .
$$

Proof. Since $X_{n_{0}}=\tilde{X}_{n_{0}}$ and the value of $\tilde{X}_{n}$ is frozen to be $\tilde{X}_{n_{0}}$ for all $n \geq n_{0}$ and $\tau\left(n_{0}\right) \geq n_{0}$, we have that

$$
\left|\tilde{X}_{n_{0}}\right|=\left|\tilde{X}_{\tau\left(n_{0}\right)}\right| \leq M_{\tau\left(n_{0}\right)} \leq Q_{\tau\left(n_{0}\right)} \leq N_{n_{0}} .
$$

Remark 4.2. If we did not switch to working with the sequence $\left(\tilde{X}_{n}\right)$, then the inequalities above would hold with $X$ in place of $\tilde{X}$ but the first equality would not hold. Freezing the value of $\tilde{X}_{n}$ at $X_{n_{0}}$ for $n \geq n_{0}$ allows us to focus exactly on bounding the quantity of interest, which is $\mathbb{E}\left[X_{n_{0}}^{2}\right]$, without worrying about fluctuations in the sequence due to $A_{n}$ 's for $n \geq n_{0}$.

\section{AnAlysis OF THE AlgORIthm}

Now we state our main theorem showing that $N_{n}$ decreases on average when large. We need to specify a filtration; we will include in the $\sigma$-algebra $\mathcal{F}_{n}$ all steps of the algorithm which have happened so far.

Definition 1. Let $\mathcal{F}_{n}$ be the $\sigma$-algebra generated by $\left(A_{k}\right)_{k<n}$, $\left(W_{k}\right)_{k<n}$ and all bits sent by the encoder in time-steps up through $n-1$.

Theorem 5.1. Assume that $A_{n}$ and $W_{n}$ have finite $(4+$ $\epsilon)$-th moments. Let $n_{0}$ be any fixed time. Then, for any 
$n<n_{0}$, positive constant $c<\min \left(1-\sigma_{A}^{2}, \frac{3}{4}\right)$ that does not depend on $n_{0}$, sufficiently large $P, M_{0}, K$, and sufficiently small $\delta=\delta\left(c, P, M_{0}, K\right)$, there exists a constant $C\left(c, P, M_{0}, K, \sigma_{W}, \sigma_{A}\right)$ such that

$$
\mathbb{E}\left[N_{n}^{2}\right] \leq C .
$$

Our main result, Theorem 1.1 follows immediately as a corollary.

Proof of Theorem 1.1. For any fixed $n_{0}$, modifying the sequence at $n_{0}$ results in a sequence $N_{n}$. We know from Prop 4.1 that $\left|X_{n_{0}}\right| \leq N_{n_{0}}$. Using Thm. 5.1 and taking expectations gives the result as:

$$
\begin{aligned}
\mathbb{E}\left[X_{n_{0}}^{2}\right] & \leq \mathbb{E}\left[N_{n_{0}}^{2}\right] \\
& =\sup _{n_{0}} \sup _{n<n_{0}} \mathbb{E}\left[N_{n}^{2}\right] \\
& <\infty .
\end{aligned}
$$

\section{A. Proof of Theorem 5.1}

For a time-step $n<n_{0}$ that is in normal mode, we have that $\tau(n)=n$. We write:

$$
N_{n+1}^{2}=N_{n+1}^{2} \mathbf{1}_{\tau(n+1)=n+1}+N_{n+1}^{2} \mathbf{1}_{\tau(n+1)>n+1} .
$$

We estimate the conditional expectation of each term separately. For the first term we establish a second-moment averaged decrease from $M_{n}$ to $M_{n+1}$ when we stay in normal mode, while for the second we show that the contributions from emergency mode are small. The first step is elementary but with slightly involved algebra, while the second requires us to control the tails for the length of a round (and thus requires a bounded $4+\epsilon$-th moment). We split this work into the following pair of lemmas.

Lemma 5.2. Suppose that time-steps $n$ and $n+1$ are both $<n_{0}$ and start in normal mode, so that $\tau(n)=n$ and $\tau(n+$ $1)=n+1$. Then for any positive constant $c<1-\sigma_{A}^{2}$, sufficiently large $K$, arbitrary $P, M_{0}$, and sufficiently small $\delta=\delta(c, K, P)$ we have

$$
\begin{aligned}
\mathbb{E}\left[N_{n+1}^{2} \mathbf{1}_{\tau(n+1)=n+1} \mid \mathcal{F}_{n}\right] & \\
\leq & (1-c) N_{n}^{2}+2 \sigma_{W}^{2}+K M_{0}^{2} \text { a.s. }
\end{aligned}
$$

Lemma 5.3. Suppose that $A$ and $W$ have finite $4+\epsilon$-th moments. Further, suppose that time-step $n$ starts in normal mode, so that $\tau(n)=n$. However, $X_{n+1}>P M_{n}$, so that $\tau(n+1)>n+1$. Then,

$$
\mathbb{E}\left[N_{n+1}^{2} \mathbf{1}_{\tau(n+1)>n+1} \mid \mathcal{F}_{n}\right] \leq \varepsilon N_{n}^{2} \text { a.s. }
$$

where $\varepsilon\left(P, M_{0}\right)$ may be made made arbitrarily small by choosing $M_{0}$ sufficiently large and then $P$ sufficiently large.

We now briefly explain how to derive Theorem 5.1 from Lemmas 5.2 and 5.3.

Proof of Theorem 5.1, using Lemmas 5.2 and 5.3. First, we consider the case when we are in normal mode and $\tau(n)=n$, and use the representation in (23). For $c$ as in the theorem statement, we first pick $P, M_{0}$ large enough such that, in the language of Lemma 5.3, $\varepsilon\left(P, M_{0}\right)$ satisfies

$$
c+\varepsilon<\min \left(1-\sigma_{A}^{2}, \frac{3}{4}\right) .
$$

Lemma 5.3 then gives:

$$
\mathbb{E}\left[N_{n+1}^{2} \mathbf{1}_{\tau(n+1)>n+1} \mid \mathcal{F}_{\tau(n)}\right] \leq \varepsilon N_{n}^{2} \text { a.s. }
$$

Then substituting $c+\varepsilon$ for $c$ in Lemma 5.2, pick $K$ large and then $\delta$ small to make

$$
\begin{aligned}
\mathbb{E}\left[N_{n+1}^{2} \mathbf{1}_{\tau(n+1)=n+1} \mid \mathcal{F}_{\tau(n)}\right] & \\
\leq & (1-c-\varepsilon) N_{n}^{2}+2 \sigma_{W}^{2}+K M_{0}^{2}
\end{aligned}
$$

hold whenever $\tau(n)=n$. Combining (27) and (28) using (23), we have

$$
\mathbb{E}\left[N_{n+1}^{2} \mid \mathcal{F}_{\tau(n)}\right] \leq(1-c) N_{n}^{2}+2 \sigma_{W}^{2}+K M_{0}^{2} \text { a.s. }
$$

in this case.

Now consider the case that we are in emergency mode and $\tau(n)>n$. Here, due to (18) we automatically have $N_{n+1}=$ $\frac{N_{n}}{2}$. Since $c<\frac{3}{4}$ and $\mathcal{F}_{\tau(n)}$ includes $N_{n}$, it follows that:

$$
\mathbb{E}\left[N_{n+1}^{2} \mid \mathcal{F}_{\tau(n)}\right]=\frac{1}{4} N_{n}^{2} \leq(1-c) N_{n}^{2} \text { a.s. }
$$

Thus (29) also holds when $\tau(n)>n$.

Let

$$
D=2 \sigma_{W}^{2}+(1+K) M_{0}^{2},
$$

and observe that (29) implies

$$
\mathbb{E}\left[N_{n+1}^{2}\right] \leq(1-c) \mathbb{E}\left[N_{n}^{2}\right]+2 \sigma_{W}^{2}+K M_{0}^{2} .
$$

Now we can proceed using induction. Assume $\mathbb{E}\left[N_{n}^{2}\right] \leq \frac{D}{c}=$ $C$. Then, we have from (32) that

$$
\begin{aligned}
\mathbb{E}\left[N_{n+1}^{2}\right] & \leq(1-c) \frac{D}{c}+D-M_{0}^{2} \\
& <\frac{D}{c},
\end{aligned}
$$

which is a constant that does not depend on $n_{0}$. It remains to verify the base case. Since $X_{0}=0$, we know that $M_{0}=I_{0}$, the initial $\tau(0)=0$ and $Q_{0}=\sqrt{(1+K) M_{0}^{2}}$. Hence $N_{0}=$ $Q_{0} \cdot 2^{0}=Q_{0}$ and $\mathbb{E}\left[N_{0}^{2}\right] \leq D \leq \frac{D}{c}$, since $c<1$.

This proves Theorem 5.1 assuming the lemmas.

\section{B. Proofs of Lemmas}

Finally, we prove the two key lemmas below.

Proof of Lemma 5.2. In the setting of Lemma 5.2, we have $\tau(n)=n$, and this implies $\mathcal{F}_{\tau(n)}=\mathcal{F}_{n}$ and $N_{n}=Q_{n}$. Further, we have $\tau(n+1)=n+1$, and so $N_{n+1}=Q_{n+1}$. Hence, for the remainder of this proof we will refer to the $Q$ 's instead of the $N$ 's. Similarly, since $n<n_{0}$ we will refer to the $X$ 's instead of the $\tilde{X}$ 's, the $A$ 's instead of $\tilde{A}$ 's, etc; these modifications are only important for Lemma 5.3 and not Lemma 5.2.

Note that from the definition (17) we have $Q_{n}=$ $\sqrt{\tilde{M}_{n}^{2}+K \tilde{I}_{n}^{2}}=\sqrt{M_{n}^{2}+K I_{n}^{2}}$. We will obtain the bound (24) 
by bounding both $M_{n}$ and $I_{n}$. We first start with obtaining a bound on $M_{n}$.

From (3) we have

$$
\mathbb{E}\left[X_{n+1}^{2} \mid \mathcal{F}_{n}\right]=\sigma_{A}^{2} X_{n}^{2}+\left(\mu_{A} X_{n}-U_{n}\right)^{2}+\sigma_{W}^{2} .
$$

In addition, since we are in normal mode we have from (9) that

$$
\left|\mu_{A} X_{n}-U_{n}\right| \leq \mu_{A} I_{n}
$$

This implies

$$
\begin{aligned}
& \mathbb{E}\left[X_{n+1}^{2} \mathbf{1}_{\tau(n+1)=n+1} \mid \mathcal{F}_{n}\right] \\
& \quad \leq E\left[X_{n+1}^{2} \mid \mathcal{F}_{n}\right] \leq \sigma_{A}^{2} M_{n}^{2}+\mu_{A}^{2} I_{n}^{2}+\sigma_{W}^{2}
\end{aligned}
$$

Furthermore, we assume this round lasts exactly one time-step and $\tau(n+1)=n+1$. Hence, we also have that either

$$
\left|M_{n+1}-X_{n+1}\right| \leq \operatorname{P} \delta M_{n},
$$

or the state $X_{n+1}$ has gotten below the lower limit $M_{0}$ and we have that $M_{n+1}=M_{0}$ :

$$
\left|X_{n+1}\right| \leq M_{n+1}=M_{0}
$$

From (37) and (38) we obtain the error estimate

$$
\begin{aligned}
& \mathbb{E}\left[\left(M_{n+1}^{2}-X_{n+1}^{2}\right) \mathbf{1}_{\tau(n+1)=n+1} \mid \mathcal{F}_{n}\right] \leq \\
& P \delta M_{n}\left(2 \mathbb{E}\left[\left|X_{n+1}\right| \mathbf{1}_{\tau(n+1)=n+1} \mid \mathcal{F}_{n}\right]+P \delta M_{n}\right) \\
& +M_{0}^{2} .
\end{aligned}
$$

This follows because we have that $\left(a^{2}-b^{2}\right)=(a+b)(a-b)$, and further we observe that $|X+M| \leq|2 X|+|M-X|$. The last term $M_{0}^{2}$ comes from the case where the state hits the lower limit, and follows from (38).

Now using the definition of $X_{n+1}$ in (1), the choice of $U_{n}$ in (8) and the simple estimate $\mathbb{E}[|Z|] \leq \mu_{Z}+\sigma_{Z}$ for any real random variable $Z$, we obtain:

$$
\begin{aligned}
& \mathbb{E}\left[\left|X_{n+1}\right| \mid \mathcal{F}_{n}\right] \\
\leq & \mathbb{E}\left[\left|A_{n}\right|\right]\left|X_{n}\right|+\mathbb{E}\left[\left|W_{n}\right|\right]+\left|\mu_{A} M_{n}\right| \\
\leq & \left(2 \mu_{A}+\sigma_{A}\right) M_{n}+\sigma_{W} .
\end{aligned}
$$

Adding (36) and (39), and then using the bound in (40) for the conditional expectation of $\left|X_{n+1}\right|$ we get that:

$$
\begin{aligned}
\mathbb{E}\left[M_{n+1}^{2} \mathbf{1}_{\tau(n+1)}=n+1\right. & \left.\mid \mathcal{F}_{n}\right] \leq \\
\left(\sigma_{A}^{2}+\right. & \left.\left(2 \mu_{A}+\sigma_{A}\right)(2 P \delta)+P^{2} \delta^{2}\right) M_{n}^{2} \\
& +2 P \delta \sigma_{W} M_{n}+\mu_{A}^{2} I_{n}^{2}+\sigma_{W}^{2}+M_{0}^{2} .
\end{aligned}
$$

Since $2 P \delta \sigma_{W} M_{n} \leq P^{2} \delta^{2} M_{n}^{2}+\sigma_{W}^{2}$ we can eliminate the term that is linear in $M_{n}$, obtaining the slightly cleaner

$$
\begin{aligned}
\mathbb{E}\left[M_{n+1}^{2} \mathbf{1}_{\tau(n+1)=n+1} \mid \mathcal{F}_{n}\right] \leq & \\
\left(\sigma_{A}^{2}+\left(2 \mu_{A}+\sigma_{A}\right)\right. & \left.2 P \delta)+2 P^{2} \delta^{2}\right) M_{n}^{2} \\
& +\mu_{A}^{2} I_{n}^{2}+2 \sigma_{W}^{2}+M_{0}^{2} .
\end{aligned}
$$

Estimating $I_{n+1}^{2}$ is much simpler; since we know from (5) that $\left|I_{n+1}\right| \leq \max \left(\frac{P \delta M_{n}}{2}, M_{0}\right)$ whenever $\tau(n+1)=n+1$ we obviously have

$$
\mathbb{E}\left[I_{n+1}^{2} \mathbf{1}_{\tau(n+1)=n+1} \mid \mathcal{F}_{n}\right] \leq P^{2} \delta^{2} M_{n}^{2}+M_{0}^{2} .
$$

Now, recall we have $Q_{n}=\sqrt{\tilde{M}_{n}^{2}+K \tilde{I}_{n}^{2}}=\sqrt{M_{n}^{2}+K I_{n}^{2}}$. Adding (42) and (43) we get:

$$
\begin{aligned}
& \mathbb{E}\left[Q_{n+1}^{2} \mathbf{1}_{\tau(n+1)=n+1} \mid \mathcal{F}_{n}\right] \leq \\
&\left(\sigma_{A}^{2}+\left(2 \mu_{A}+\sigma_{A}\right)(2 P \delta)+(2+K) P^{2} \delta^{2}\right) M_{n}^{2} \\
&+\mu_{A}^{2} I_{n}^{2}+2 \sigma_{W}^{2}+K M_{0}^{2}
\end{aligned}
$$

Now, we choose parameters $K, \delta$ so that we can bound the coefficients of $M_{n}^{2}$ and $I_{n}^{2}$ in (44), i.e. so that the following two inequalities hold:

$$
\sigma_{A}^{2}+\left(2 \mu_{A}+\sigma_{A}\right)(2 P \delta)+(2+K) P^{2} \delta^{2} \leq 1-c,
$$

and

$$
\mu_{A}^{2} \leq(1-c) K
$$

These can be satisfied for any positive $c<1-\sigma_{A}^{2}$ if we take $P$ arbitrary, choose $K \geq \frac{\mu_{A}^{2}}{1-c}$, and finally take $\delta=\delta(c, K, P)$ sufficiently small. This gives:

$$
\begin{aligned}
& \mathbb{E}\left[Q_{n+1}^{2} \mathbf{1}_{\tau(n+1)=n+1} \mid \mathcal{F}_{n}\right] \\
& \leq(1-c) M_{n}^{2}+(1-c) K I_{n}^{2}+2 \sigma_{W}^{2}+(K) M_{0}^{2} \\
& =(1-c) Q_{n}^{2}+2 \sigma_{W}^{2}+(K) M_{0}^{2} .
\end{aligned}
$$

Remark 5.4. Making the choice $c<1-\sigma_{A}^{2}$ is technically the only place in the proof where we use the necessary assumption $\sigma_{A}^{2}<1$. The value for $P$ was essentially irrelevant in the above calculations but picking $P$ appropriately will be important for Lemma 5.3. In particular, it is important that an arbitrarily large value of $P$ is acceptable if we take $\delta$ very small to compensate. The value $M_{0}$ did not come into play but will play a minor role in proving Lemma 5.3.

For some intuition on the definition of $Q_{n}$, note that if $M_{n}=I_{n}$, i.e. when we hit the lower limit of $M_{0}$, then we cannot guarantee an averaged squared decrease of $M_{n} \rightarrow M_{n+1}$. However, in this case we expect $I_{n+1}=P \delta I_{n}$ to hold. In the other extreme case when we know $X_{n}=M_{n}$, we expect $M_{n} \rightarrow M_{n+1}$ to result in a decrease, and so picking $\delta$ small enough ensures that the regularization term $K I_{n}^{2}$ does not hurt us too much. Hence in both cases we expect a squared decrease from $Q_{n} \rightarrow Q_{n+1}$ when parameters are chosen appropriately, e.g. as above.

Proof of Lemma 5.3. Note that $\tau(n)=n$, and we estimate $\mathbb{E}\left[N_{n+1}^{2} \mathbf{1}_{\tau(n+1)>n+1}\right]$ under this assumption. If $\tau(n+1)=$ $n+k+1$ (for $k \geq 1$ ) then we have

$$
\begin{aligned}
N_{n+1}^{2} & \leq 2^{2 k}\left(\tilde{M}_{n+k+1}^{2}+K \tilde{I}_{n+k+1}^{2}\right) \\
& \leq 2^{2 k}(1+K) \tilde{M}_{n+k+1}^{2} \\
& =2^{2 k}(1+K) P^{2 k+2} \tilde{M}_{n}^{2} \\
& \leq 2^{2 k}(1+K) P^{2 k+2} N_{n}^{2} .
\end{aligned}
$$

To control (52), it will suffice to show that $k=\tau(n+1)-$ $(n+1)$ has very fast decaying tails. This is what we will do. 
We have $\tilde{X}_{n+1}=\tilde{A}_{n} \tilde{X}_{n}+\tilde{W}_{n}-\tilde{U}_{n}$. For later emergency rounds, we have $\tilde{U}_{n+j}=0$ and so

$$
\tilde{X}_{n+j+1}=\tilde{A}_{n+j} \tilde{X}_{n+j}+\tilde{W}_{n+j} .
$$

Hence again taking $\tau(n+1)=n+k+1$, for each $k \geq h \geq 0$ we may write

$$
\begin{array}{r}
\tilde{X}_{n+h+1}=\left(\tilde{A}_{n+1} \tilde{A}_{n+2} \ldots \tilde{A}_{n+h}\right)\left(\tilde{A}_{n} \tilde{X}_{n}-\tilde{U}_{n}\right) \\
+\sum_{i=0}^{h}\left(\tilde{W}_{i} \prod_{j=i+1}^{h} \tilde{A}_{n+j}\right) .
\end{array}
$$

Since (54) only holds when $\tau(n+1) \geq n+h+1$, for each $h \geq 0$ we define

$$
\begin{aligned}
Z_{n+h+1} \triangleq\left(\tilde{A}_{n+1} \tilde{A}_{n+2} \ldots \tilde{A}_{n+h}\right)\left(\tilde{A}_{n} \tilde{X}_{n}-\tilde{U}_{n}\right) \\
+\sum_{i=0}^{h}\left(\tilde{W}_{i} \prod_{j=i+1}^{h} \tilde{A}_{n+j}\right) .
\end{aligned}
$$

We have that $Z_{n+h+1}=\tilde{X}_{n+h+1}$ when $\tau(n+1) \geq n+h+1$ for $h \geq 0$. Now, we can upper bound the absolute value of this. Recall that ${ }_{\tilde{U}}\left|\tilde{U}_{n}\right| \leq\left|\mu_{A}\right| \tilde{M}_{n}$, and since $\tau(n)=n$ we have also $\left|\tilde{X}_{n}\right| \leq \tilde{M}_{n}$. Therefore we obtain:

$$
\begin{aligned}
\left|Z_{n+h+1}\right| \leq \tilde{M}_{n}\left(\left|\tilde{A}_{n}\right|\right. & \left.+\left|\mu_{A}\right|\right)\left(\left|\tilde{A}_{n+1} \tilde{A}_{n+2} \ldots \tilde{A}_{n+h}\right|\right) \\
& +\sum_{i=0}^{h}\left(\left|\tilde{W}_{i}\right| \prod_{j=i+1}^{h}\left|\tilde{A}_{n+j}\right|\right) .
\end{aligned}
$$

The event $\tau(n+1)=n+k+1$ implies $\left|Z_{n+k}\right| \geq P^{k} \tilde{M}_{n}$ which is an abnormally large value. To control the probability that $\tau(n+1)=n+k+1$, we will estimate the $\alpha$-moments of $Z_{n+h+1}$. To do this we need to control the moments for each term in (56). For convenience, define

$$
m_{\alpha} \triangleq \max \left(1, \mathbb{E}\left[\left(|A|+\left|\mu_{A}\right|\right)^{\alpha}\right]\right),
$$

and

$$
\ell_{\alpha} \triangleq \mathbb{E}\left[|W|^{\alpha}\right] .
$$

Some of the $\tilde{A}_{n+j}$ terms may have the law of $A$, while others may be almost surely 1 . However, the value $m_{\alpha}$ will serve to estimate both cases uniformly; similarly $W$ might be identically 0 , and this will also be fine. We have the simple following estimate for the first term in (56), which follows from (57), the independence of $\tilde{M}_{n}$ and all of the $\tilde{A}_{k}$ 's, which are i.i.d.:

$$
\begin{array}{r}
\mathbb{E}\left[\left|\tilde{M}_{n}\left(\left|\tilde{A}_{n}\right|+\left|\mu_{A}\right|\right)\left(\tilde{A}_{n+1} \tilde{A}_{n+2} \ldots \tilde{A}_{n+h}\right)\right|^{\alpha} \mid \mathcal{F}_{n}\right] \\
\leq \tilde{M}_{n}^{\alpha} m_{\alpha}^{h+1} .
\end{array}
$$

For the individual terms in the summation in (56), we similarly have from (57), (58):

$$
\left\|B_{i}\right\|_{\alpha}^{\alpha} \leq \ell_{\alpha} m_{\alpha}^{h-i}
$$

where

$$
B_{i} \triangleq\left(\left|\tilde{W}_{i}\right| \prod_{j=i+1}^{h}\left|\tilde{A}_{n+j}\right|\right),
$$

and the $L_{\alpha}$-norm is taken with respect to $\mathbb{E}\left(\cdot \mid \mathcal{F}_{n}\right)$. The subadditivity of the $L_{\alpha}$-norm implies that

$$
\left\|\sum_{i} B_{i}\right\|_{\alpha}^{\alpha} \leq\left(\sum_{i}\left\|B_{i}\right\|_{\alpha}\right)^{\alpha} .
$$

This gives us the following bound for some constant $C(\alpha)$, where we also use $m_{\alpha}^{h-i} \leq m_{\alpha}^{h}$ :

$$
\mathbb{E}\left[\left(\sum_{i=0}^{h} B_{i}\right)^{\alpha} \mid \mathcal{F}_{n}\right] \leq C(\alpha) \ell_{\alpha} m_{\alpha}^{h} .
$$

To combine the two estimates (59) and (63) we simply note that

$$
|x+y|^{\alpha} \leq 2^{\alpha}\left(|x|^{\alpha}+|y|^{\alpha}\right)
$$

for all reals $x, y$. Hence we obtain the following, where $C_{1}(\alpha)$ and $C_{2}(\alpha)$ are also constants:

$$
\begin{aligned}
\mathbb{E}\left[\left|Z_{n+h+1}\right|^{\alpha} \mid \mathcal{F}_{n}\right] & \leq C_{1}(\alpha)\left(\tilde{M}_{n}^{\alpha} m_{\alpha}^{h+1}+\ell_{\alpha} m_{\alpha}^{h}\right) \\
& \leq C_{2}(\alpha) m_{\alpha}^{h}\left(\tilde{M}_{n}^{\alpha}+1\right) .
\end{aligned}
$$

Because we have $\left|\tilde{M}_{n}\right| \geq M_{0}$ for all $n$, we may simply say for a constant $C_{3}(\alpha)$ :

$$
\mathbb{E}\left[\left|Z_{n+h+1}\right|^{\alpha} \mid \mathcal{F}_{n}\right] \leq C_{3}(\alpha) m_{\alpha}^{h} \tilde{M}_{n}^{\alpha} .
$$

Now, in the event that $\tau(n+1)=n+k+1$ we must have

$$
\left|Z_{n+k+1}\right| \geq P^{k+1} \tilde{M}_{n}
$$

Therefore by the Markov inequality and (66),

$$
\begin{aligned}
& \mathbb{P}\left[\left|Z_{n+k+1}\right| \geq P^{k+1} \tilde{M}_{n} \mid \mathcal{F}_{n}\right] \\
& \leq P^{-(k+1) \alpha} \tilde{M}_{n}^{-\alpha} \mathbb{E}\left[\left|Z_{n+k+1}\right|^{\alpha} \mid \mathcal{F}_{n}\right] \\
& \leq C_{3}(\alpha) P^{-(k+1) \alpha} m_{\alpha}^{k+1} .
\end{aligned}
$$

This gives desired bound on $\mathbb{E}\left[\left|N_{n+1}^{2} \mathbf{1}_{\tau(n+1)>n+1}\right| \mathcal{F}_{n}\right]$. We sum over $k$ to calculate the expectation over different values of $\tau(n+1)$, and the probability of $\tau(n+1)=n+k+1$ is given by the LHS of (68).

$$
\begin{aligned}
& \mathbb{E}\left[\left|N_{n+1}^{2} \mathbf{1}_{\tau(n+1)>n+1}\right| \mathcal{F}_{n}\right] \\
& \leq \sum_{k \geq 1}\left(2^{2 k}(K+1) P^{2 k+2} N_{n}^{2} \mathbb{P}\left[\left|Z_{n+k}\right| \geq P^{k} \tilde{M}_{n}\right]\right) \\
& \leq C_{4}(\alpha) N_{n}^{2} \sum_{k \geq 1}\left(2^{2 k-2} P^{2 k+2-k \alpha} \tilde{m}_{\alpha}^{k}\right),
\end{aligned}
$$

for a fourth constant $C_{4}(\alpha)$. The first inequality (69) follows from (68), and the second (70) follows from (52). This sum is a geometric series with first term $P^{4-\alpha} m_{\alpha}$ and ratio $4 P^{2-\alpha} \tilde{m}_{\alpha}$. Hence we obtain

$$
\begin{aligned}
& \mathbb{E}\left[N_{n+1}^{2} \mathbf{1}_{\tau(n+1)>n+1} \mid \mathcal{F}_{n}\right] \leq \\
& C_{\alpha}^{(4)} N_{n}^{2}\left(\frac{P^{4-\alpha} m_{\alpha}}{1-4 P^{2-\alpha} \tilde{m}_{\alpha}}\right) .
\end{aligned}
$$

Since the analysis so far has been uniform in $P$, we see that for any $\alpha>4$, taking $P$ sufficiently large gives the upper bound

$$
\mathbb{E}\left[N_{n+1}^{2} \mathbf{1}_{\tau(n+1)>n+1} \mid \mathcal{F}_{n}\right] \leq \varepsilon N_{n}^{2} .
$$

as desired. (Recall $A, W$ have bounded $\alpha$-moment for some $\alpha>4$.) 


\section{CONCLUSION AND FUTURE WORK}

Our paper considered the problem of stabilizing a system that was growing unpredictably using observations over a ratelimited channel. We provide a time-varying strategy that is able to stabilize the system. The controller takes different actions based on whether the value of the system state is in a predicted interval or not - we believe such a time-varying strategy is essential for this problem. In future work, we aim to close the gap between this strategy and the converse bound in [6]. Extensions to the vector case are also interesting, since the differential growth rate along different directions must be taken into account. The $A_{n}$ 's can also be thought of as multiplicative i.i.d. random noise, so we hope that this work can help in understanding rate-limited control for this special class of nonlinear stochastic systems. It would be interesting to see if the techniques can be extended to understand a multiplicative control input in superposition with the multiplicative noise (as well as an additive control).

\section{ACKNOWLEDGMENTS}

We thank Miklós Rácz and Serdar Yüksel for interesting discussions regarding this problem. We are very grateful to the reviewers whose feedback has greatly improved the presentation of this work, as well as to the reviewers of the ISIT paper. The work of Victoria Kostina was supported in part by the National Science Foundation (NSF) under grant CCF1751356, and both she and Gireeja Ranade were supported by the Simons Institute for the Theory of Computing. The authors would also like to thank Microsoft Research, where part of the work was performed.

\section{REFERENCES}

[1] V. Kostina, Y. Peres, G. Ranade, and M. Sellke, "Stabilizing a system with an unbounded random gain using only finitely many bits," in 2018 IEEE International Symposium on Information Theory (ISIT). IEEE, 2018, pp. 2256-2260.

[2] W. S. Wong and R. W. Brockett, "Systems with Finite Communication Bandwidth Constraints-Part I: State Estimation Problems," IEEE Transactions on Automatic Control, vol. 42, no. 9, pp. 1294-1299, 1997.

[3] S. Tatikonda and S. Mitter, "Control under communication constraints," IEEE Transactions on Automatic Control, vol. 49, no. 7, pp. 1056-1068, 2004.

[4] G. N. Nair and R. J. Evans, "Stabilization with data-rate-limited feedback: tightest attainable bounds," Systems \& Control Letters., vol. 41, no. 1 , pp. 49-56, 2000.

[5] G. N. Nair, F. Fagnani, S. Zampieri, and R. J. Evans, "Feedback control under data rate constraints: An overview," Proceedings of the IEEE, vol. 95, no. 1, pp. 108-137, 2007.

[6] V. Kostina, Y. Peres, M. Rácz, and G. Ranade, "Rate-limited control of systems with uncertain gain," in Proceedings 54th Allerton Conference on Communication, Control, and Computing, Monticello, IL, Oct. 2016, pp. 1189-1196.

[7] M. Athans, R. Ku, and S. Gershwin, "The uncertainty threshold principle: Some fundamental limitations of optimal decision making under dynamic uncertainty," IEEE Transactions on Automatic Control, vol. 22, no. 3, pp. 491-495, 1977.

[8] N. Martins, M. Dahleh, and N. Elia, "Feedback Stabilization of Uncertain Systems in the Presence of a Direct Link," IEEE Trans. Autom. Control, vol. 51, no. 3, pp. 438-447, 2006.

[9] K. Okano and H. Ishii, "Minimum data rate for stabilization of linear systems with parametric uncertainties," 2014, arXiv preprint.

[10] S. Yuksel and T. Basar, Stochastic networked control systems. Springer, 2013.

[11] S. Fang, J. Chen, and H. Ishii, Towards Integrating Control and Information Theories. Springer, 2016.
[12] P. Minero, M. Franceschetti, S. Dey, and G. N. Nair, "Data Rate Theorem for Stabilization Over Time-Varying Feedback Channels," IEEE Transactions on Automatic Control, vol. 54, no. 2, pp. 243-255, 2009.

[13] S. Yüksel, "Stochastic stabilization of noisy linear systems with fixedrate limited feedback," IEEE Transactions on Automatic Control, vol. 55, no. 12 , pp. 2847-2853, 2010.

[14] K. You and L. Xie, "Minimum data rate for mean square stabilization of discrete lti systems over lossy channels," IEEE Transactions on Automatic Control, vol. 55, no. 10, pp. 2373-2378, 2010.

[15] V. Kostina and B. Hassibi, "Rate-cost tradeoffs in control," IEEE Transactions on Automatic Control, to appear, 2019.

[16] A. S. Matveev and A. V. Savkin, Estimation and control over communication networks. Springer Science \& Business Media, 2009.

[17] K. You and L. Xie, "Minimum data rate for mean square stabilizability of linear systems with Markovian packet losses," IEEE Transactions on Automatic Control, vol. 56, no. 4, pp. 772-785, 2011.

[18] P. Minero, L. Coviello, and M. Franceschetti, "Stabilization over Markov feedback channels: the general case," IEEE Transactions on Automatic Control, vol. 58, no. 2, pp. 349-362, 2013.

[19] B. Sinopoli, L. Schenato, M. Franceschetti, K. Poolla, M. I. Jordan, and S. S. Sastry, "Kalman filtering with intermittent observations," Automatic Control, IEEE Transactions on., vol. 49, no. 9, pp. 1453-1464, 2004.

[20] S. Park and A. Sahai, "Intermittent Kalman filtering: Eigenvalue cycles and nonuniform sampling," in American Control Conference (ACC)., 2011, pp. 3692-3697.

[21] A. Chiuso, N. Laurenti, L. Schenato, and A. Zanella, "LQG-like control of scalar systems over communication channels: The role of data losses, delays and SNR limitations," Automatica, vol. 50, no. 12, pp. 31553163, 2014.

[22] S. Dey, A. Chiuso, and L. Schenato, "Feedback Control Over Lossy SNR-Limited Channels: Linear Encoder-Decoder-Controller Design," IEEE Transactions on Automatic Control, vol. 62, no. 6, pp. 3054-3061, 2017.

[23] L. Schenato, B. Sinopoli, M. Franceschetti, K. Poolla, and S. S. Sastry, "Foundations of control and estimation over lossy networks," Proceedings of the IEEE, vol. 95, no. 1, pp. 163-187, 2007.

[24] J. P. Hespanha, P. Naghshtabrizi, and Y. Xu, "A survey of recent results in networked control systems," Proceedings of the IEEE, vol. 95, no. 1, pp. 138-162, 2007.

[25] V. N. Phat, J. Jiang, A. V. Savkin, and I. R. Petersen, "Robust stabilization of linear uncertain discrete-time systems via a limited capacity communication channel," Systems \& Control Letters, vol. 53, no. 5, pp. $347-360,2004$

[26] K. Okano and H. Ishii, "Data rate limitations for stabilization of uncertain systems," in 51st IEEE Conference on Decision and Control, Maui, Hawaii, Dec. 2012, pp. 3286-3291.

[27] _ "Data Rate Limitations for Stabilization of Uncertain Systems over Lossy Channels," in American Control Conference, Montreal, Canada, June 2012, pp. 1260-1265.

[28] G. Nair and R. Evans, "Stabilizability of Stochastic Linear Systems with Finite Feedback Data Rates," SIAM Journal on Control and Optimization., vol. 43, no. 2, pp. 413-436, 2004.

[29] R. W. Brockett and D. Liberzon, "Quantized feedback stabilization of linear systems," IEEE Transactions on Automatic Control, vol. 45, no. 7 , pp. 1279-1289, 2000.

[30] S. Yuksel and S. P. Meyn, "Random-time, state-dependent stochastic drift for markov chains and application to stochastic stabilization over erasure channels," IEEE Transactions on Automatic Control, vol. 58, no. 1, pp. 47-59, 2013.

[31] V. Kostina, Y. Peres, G. Ranade, and M. Sellke, "Exact minimum number of bits to stabilize a linear system," in Proceedings 57th IEEE Conference on Decision and Control, Miami, CA, Dec. 2018.

[32] M. Fu and L. Xie, "Quantized feedback control for linear uncertain systems," International Journal of Robust and Nonlinear Control, vol. 20 , no. 8 , pp. $843-857,2010$

[33] K. Zhou and J. C. Doyle, Essentials of robust control. Prentice hall Upper Saddle River, NJ, 1998.

[34] G. Ranade and A. Sahai, "Control capacity," IEEE Transactions on Information Theory, vol. 65, no. 1, pp. 235-254, 2018.

[35] _ "Non-coherence in estimation and control," in 51st Annual Allerton Conf. on Comm., Control, and Comp., 2013.

[36] J. Ding, Y. Peres, and G. Ranade, "A tiger by the tail: when multiplicative noise stymies control," in IEEE International Symposium on Information Theory, Barcelona, Spain, July 2016. 